\title{
Treating Coccydynia Using Primal Reflex Release Technique TM Case Report
}

\section{Carter $\mathrm{C}^{*}$}

Department of physiotherapy, Alabama State University, USA

*Corresponding author: Cleve Carter, Department of physiotherapy, Alabama State University, ASU-915 S. Jackson Street, Montgomery, AL 36104 Dove PT-445 Dexter Avenue, Suite 4050, Montgomery, AL 36104. USA, Tel: 334-229-1045; 334-549-4231; Email: ccarter@alasu.edu

\section{Case Report}

Volume 3 Issue 1

Received Date: January 16, 2020

Published Date: January 29, 2020

\section{Abstract}

Background and Purpose: The hallmark presentation of coccydynia is localized pain over the coccyx. Patients typically report having tailbone pain; yet, the exact etiology of coccydynia is unknown. Factors such as obesity and female gender increase the risk of developing coccydynia with women being 5 times more likely to develop coccydynia than men. The purpose of this case report is to share the outcomes (results) of using Primal Reflex Release TechniqueTM (PRRTTM) to treat coccydynia.

Method and Procedures: A 34-year-old Euro-American female patient presented to physical therapy with a complaint of recurrent, chronic tailbone pain with prolonged sitting on hard surfaces and surfaces with mild cushion.To determine the effects of the treatments, the patient was examined using the following tests and measures and outcome measurement tools: The 1 Minute NocioceptivExamTM, Two-Minute Walk Test (2MWT), Chair Sit and Reach Test (CSRT), the Numeric Pain Rating Scale (NPRS), the Modified Oswestry Low Back Pain Disability Questionnaire, and the Global Rating of Change (GRoC) Scale.

Results: On discharge assessment (6th visit), the patient reported no $(0 / 10)$ tenderness and pain during The 1 Minute NocioceptivExamTM with coccyx palpation and sidebending mobilizations. The PRRTTM coccyx release techniques decreased the patient's worst coccyx pain to $1 / 10$ with prolonged sitting after 1 hour on the NPRS at discharge versus $8 / 10$ worst coccyx pain with prolonged sitting after 1 hour on the NPRS at initial examination.

Discussion: The results support the effectiveness of using PRRT to treat coccyx pain. Using PRRTTM, an intervention model aimed at treating an up-regulated autonomic nervous system, may have contributed to the positive patient outcomes.

Conclusion: The results showed that the PRRTTM paradigm's coccyx release techniques can be effective in treating a case of coccydynia if not some or most cases. The results are not generalizable to all patients who may present to physical therapy with a complaint of coccyx pain including the male sex. Further research could include a large, diverse sample size.

Keywords: Coccydynia; Reflex; Technique TM 


\section{Annals of Physiotherapy \& Occupational Therapy}

\section{Background and Purpose}

The hallmark presentation of coccydynia is localized pain over the coccyx [1]. Patients typically report having tailbone pain; yet, the exact etiology of coccydynia is unknown [1]. Factors such as obesity and female gender increase the risk of developing coccydynia with women being 5 times more likely to develop coccydynia than men [2]. External or internal trauma is the most common cause of coccydynia [1]. Major trauma usually occurs due to a backwards fall, but minor trauma usually occurs due to prolonged or repetitive sitting on hard, narrow surfaces [3,4]. A backwards fall is usually the cause of coccydynia resulting in an ecchymosed, fractured, or dislocated coccyx [3]. Prolonged or repetitive sitting on solid, narrow, or uncomfortable surfaces can result in minor trauma to the coccyx and surrounding structures [4]. The pain is normally exacerbated with prolonged sitting, trunk backward bending while seated, prolonged standing, and standing from the sitting position [1]. Coccydynia may also be exacerbated with sexual intercourse or defecation [1].

Subjective history can reveal trauma with an acute onset of pain or chronic onset of pain or can reveal insidious pain without transparent etiology [1]. Physical examination may cause the patient to present with a nocioceptive (startle or withdrawal) response with palpation or mobilization of the coccyx [1]. In addition, mobilization or manipulation may cause pain and bare hypomobility or hypermobility of the sacrococcygeal joint [1]. Other causes of coccyx pain, such as tumors, fractures, dislocations, spasms, and masses, should be ruled out [1]. Radiographic imaging can be performed to determine the presence of arthritic changes, nodules, or fractures [4]. Dynamic x-ray and magnetic resonance imaging can help assess sacrococcygeal hypomobility or hypermobility [4].

There are various conservative treatment options for coccydynia. Conservative treatment is successful in most cases, and many cases improve without medical intervention [5-7]. More aggressive treatments may be warranted in cases that do not respond to conservative treatments [1]. Pelvic floor rehabilitation can be beneficial for coccydynia in cases related to pelvic floor muscle hypertonicity. ${ }^{1}$ Not only can manual therapy be diagnostic, but also it can be therapeutic [1]. Manual therapy can help correct or eliminate associated soft tissue dysfunctions that cause pain [8]. The use of transcutaneous electrical nerve stimulation (TENS) can help manage pain and can be therapeutic [9].

The purpose of this case report is to share the outcomes (results) of using Primal Reflex Release Technique ${ }^{\mathrm{TM}}$ (PRRT ${ }^{\mathrm{TM}}$ ) to treat coccydynia. Evidence-based literature supporting the effectiveness of PRRT ${ }^{\mathrm{TM}}$ interventional procedures is lacking.
However, licensed clinicians such as physical therapists have testified to the effectiveness and positive results (outcomes) that patients experience with the use of PRRT ${ }^{\mathrm{TM}}$. In addition, there are numerous positive patient testimonials. PRRT ${ }^{\mathrm{TM}}$ is a treatment approach to patient care that involves downregulating a hyper-stimulated sympathetic nervous system or "up-regulated" autonomic nervous system in order to reduce patterns of pain [10]. The model is intended to influence the nervous system by rebooting (resetting) hyperactive primal reflexes within the body. ${ }^{10}$ Trigger Regions ${ }^{\mathrm{TM}}$ are identified using bilateral palpation (strumming) during The 1 Minute Nocioceptiv Exam ${ }^{\mathrm{TM}}[10]$. However, if a patient reports or pinpoints a specific, localized condition that has a PRRT ${ }^{\mathrm{TM}}$ designed to treat the specific, localized condition, the clinician can treat the patient using the PRRT ${ }^{\mathrm{TM}}$ designed to treat the specific condition. The clinician re-assesses the patient for an eliminated or decrease nocioceptive (startle or withdrawal) response after the PRRT is performed. The technique is considered effective if the patient has an eliminated or decreased nocioceptive (startle or withdrawal) response with palpation or movement after the PRRT ${ }^{\mathrm{TM}}$ is performed. The patient may or may not experience long-term relief and may or may not require more treatment sessions after the first initial treatment session.

\section{Methods and Procedures}

A 34-year-old Euro-American female patient presented to physical therapy with a complaint of recurrent, chronic tailbone pain with prolonged sitting on hard surfaces and surfaces with mild cushion. The patient reported that she did not experience tailbone pain with standing, walking, jogging, running, general physical activity, or exercise. The clinician explained the examination, evaluation, and plan of care process. The clinician also obtained written and verbal permission to use gathered data to write a case report or case study, and the patient signed an informed consent form. To determine the effects of the treatments, the patient was examined using the following tests and measures and outcome measurement tools: The 1 Minute Nocioceptiv Exam $^{\mathrm{TM}}$, Two-Minute Walk Test (2MWT), Chair Sit and Reach Test (CSRT), the Numeric Pain Rating Scale (NPRS), the Modified Oswestry Low Back Pain Disability Questionnaire, and the Global Rating of Change (GRoC) Scale.

On the initial examination, the patient reported marked $(7 / 10)$ tenderness and pain during The 1 Minute NocioceptivExam ${ }^{\mathrm{TM}}$ with coccyx palpation and side bending mobilizations. The patient also presented with a nocioceptive (startle) response with coccyx palpation during The 1 Minute NocioceptivExam ${ }^{\mathrm{TM}}$. The patient reported greater pain on the right with coccyx palpation and side bending mobilization. The patient reported no $(0 / 10)$ pain during and after performance of the 2MWT and CSRT on the initial 


\section{Annals of Physiotherapy \& Occupational Therapy}

examination. The patient reported $8 / 10$ for worst pain within the past two weeks with prolonged sitting after 1 hour, $0 / 10$ for best pain within the past two weeks, and $0 / 10$ for current pain on the NPRS on the initial examination. The patient's Modified Oswestry Low Back Pain Disability Questionnaire score was $8 \%$ on the initial examination. The GRoC Scale was not administered on the initial examination; however, it was administered on the 6th (discharge assessment) session. The patient attended physical therapy sessions 2 times per week for 3 weeks.

Prior to performing and using the $\mathrm{PRRT}^{\mathrm{TM}}$ interventions in this case, the physical therapist studied and practiced the PRRT $^{\mathrm{TM}}$ Home Study Course for at least 135 hours and the PRRT $^{\mathrm{TM}}$ Reflex Speed Pain Release Techniques on Digital Versatile Disc (DVD) for at least 135 hours. The physical therapist also attended the PRRT ${ }^{\mathrm{TM}}$ Level 1 Basic Course. The physical therapist performed two different coccyx release techniques from the PRRT ${ }^{\mathrm{TM}}$ paradigm each session. The first coccyx release technique entailed the patient lying prone and performing a gluteal set while the physical therapist karate chopped the gluteal musculature bilaterally from the sacrum to the coccyx. The first coccyx release technique was performed 10 times for duration of 30 seconds each treatment session. The second coccyx release technique entailed the patient lying supine with the hips and knees positioned at 90 degrees while moving the less involved left thigh across the body towards the right shoulder; the patient performed a contract-relax several times with intention to fire the lowest gluteus maximus muscle fibers. The patient performed a maximal muscle contraction for 10 seconds against manual force applied in a diagonal of hip flexionadduction. The patient relaxed for 30 seconds. Thus, the contract-relax ratio was 10:30. The second coccyx release technique was performed at 1 set of 6 repetitions of contractrelax each treatment session.

\section{Results}

On discharge assessment (6th visit), the patient reported no $(0 / 10)$ tenderness and pain during The 1 Minute NocioceptivExam ${ }^{\mathrm{TM}}$ with coccyx palpation and sidebending mobilizations. The patient also presented without a nocioceptive (startle) response with coccyx palpation during The 1 Minute NocioceptivExam ${ }^{\mathrm{TM}}$. The patient reported that she had no pain on either side with coccyx palpation and side bending mobilization. The patient reported no $(0 / 10)$ pain during and after performance of the 2MWT and CSRT on the discharge assessment. The patient reported $1 / 10$ for worst pain within the past week with prolonged sitting after 1 hour, $0 / 10$ for best pain, and $0 / 10$ for current pain on the NPRS on the discharge assessment (6th visit). The patient's Modified Oswestry Low Back Pain Disability Questionnaire score was $2 \%$ on the discharge assessment. On the GRoC Scale, the patient reported, "A very great deal better with overall functional performance with Functional Activities of Daily Living (FADL) from the time physical therapy began until now" on the discharge assessment (6th visit). In regards to her experience and outcomes, the patient also testified, "Dr. Carter was always so kind and explained every technique he was going to use to help eliminate my tailbone pain! I have seen a remarkable amount of improvement and couldn't be happier with my care and results."

\section{Discussion}

The results support the effectiveness of using PRRT to treat coccyx pain. Using PRRT ${ }^{\mathrm{TM}}$, an intervention model aimed at treating an up-regulated autonomic nervous system, may have contributed to the positive patient outcomes $[11,12]$. Not assessing and addressing the nervous system involvement could result in missing neural driven pain which could lead to chronic pain or referred pain. In turn, chronic tailbone pain or referred pain can easily result in functional limitations with prolonged sitting, standing, or walking. In coccydynia cases, symptoms are often localized to the coccyx. One must understand that the coccyx has several important functions despite its small size [1]. The coccyx is an insertion site for several muscles, ligaments, and tendons. It also functions as one leg of the tripod along with the weight-bearing ischial tuberosities in the seated position [1]. As indicated earlier, backwards leaning in a seated position increases the load on the coccyx [1]. The coccyx also positionally supports the anus [1]. Thus, the size, structure, and many functions of the coccyx predispose the coccyx to injury resulting from a fall and repetitive prolonged sitting on hard surfaces. A backward fall or repetitive prolonged sitting on hard surfaces can result not only in soft tissue damage but also in an active nocioceptive (startle or withdrawal) reflex contributing to central sensitization or an up-regulated autonomic nervous system. An up-regulated autonomic nervous system creates clinical manifestations where the PRRT ${ }^{\mathrm{TM}}$ can be used as an interventional tool to assess and treat the cause of the problem (neural driven pain) $[11,12]$.

\section{Conclusion}

The results showed that the PRRT ${ }^{\mathrm{TM}}$ paradigm's coccyx release techniques can be effective in treating a case of coccydynia if not some or most cases. The PRRT ${ }^{\mathrm{TM}}$ coccyx release techniques decreased the patients' worst coccyx pain to $1 / 10$ with prolonged sitting after 1 hour on the NPRS at discharge versus $8 / 10$ worst coccyx pain with prolonged sitting after 1 hour on the NPRS at initial examination. Therefore, the patient's coccyx pain significantly decreased with prolonged sitting using PRRT ${ }^{\mathrm{TM}}$ without the use of orthodox therapeutic exercises or modalities. The results are not generalizable to all patients who may present to physical 


\section{Annals of Physiotherapy \& Occupational Therapy}

therapy with a complaint of coccyx pain including the male sex. Future research could include assessing subjects using the PRRT ${ }^{\mathrm{TM}} 1$ Minute Nocioceptiv Exam ${ }^{\mathrm{TM}}$ and treating all areas that elicit a nocioceptive (startle or withdrawal) response to observe if less time is needed to reach functional pain free activity in subjects presenting with coccyx pain. In addition, further research could include a large, diverse sample size.

\section{References}

1. Lirette LS, Chaiban G, Tolba R, Eissa H (2014) Coccydynia: an overview of the anatomy, etiology, and treatment of coccyx pain. The Ochsner J 14(1): 84-87.

2. Maigne JY, Doursounian L, Chatellier G (2000) Causes and mechanisms of common coccydynia: role of body mass index and coccygeal trauma. Spine (Phila Pa 1976) 25(23): 3072-3079.

3. Schapiro S (1950) Low back and rectal pain from an orthopedic and proctologic viewpoint; with a review of 180 cases. Am J Surg 79(1): 117-128.

4. Pennekamp PH, Kraft CN, Stutz A, Wallny T, Schmitt O, et al. (2005) Coccygectomy for coccygodynia: does pathogenesis matter? J Trauma. 59(6): 1414-1419.

5. Thiele GH (1963) Coccygodynia: cause and treatment.
Dis Colon Rectum 6(6): 422-436.

6. Capar B, Akpinar N, Kutluay E, Mujde S, Turan A, et al. (2007) Coccygectomy in patients with coccydynia. Acta Orthop Traumatol Turc 41(4); 277-280.

7. Trollegaard AM, Aarby NS, Hellberg S (2010) Coccygectomy: an effective treatment option for chronic coccydynia: retrospective results in 41 consecutive patients. J Bone Joint Surg Br 92(2): 242-245.

8. Maigne JY, Chatellier G (2001) Comparison of three manual coccydynia treatments: a pilot study. Spine (Phila Pa 1976) 26(20): E479-E483.

9. Nathan ST, Fisher BE, Roberts CS (2010) Coccydynia: a review of pathoanatomy, aetiology, treatment and outcome. J Bone Joint Surg Br 92(12):1622-1627.

10. Hansberger BL, Baker RT, Nasypany A (2015) A novel approach to treating plantar fasciitis-effects of primal reflex release technique: a case series. Int J Sports Phys Ther 10(5): 690-699.

11. Iams JF (2005) When reflexes rule: a new paradigm in understanding why some patients don't get well. Advance for Physical Therapy and Rehab Medicine 16(3): 41.

12. Iams JF (2014) Reflexes rules and can be cruel. Curr Orthop Pract 15: 41-42. 